\title{
Are short sequence repeats (SSRs) pinpoint evolution hotspots? Cyprinidae fish case study
}

Orlov M.A. ${ }^{1 *}$, Tykhonov A.Yu. ${ }^{2}$

${ }^{I}$ Institute of Cell Biophysics of RAS, Poushchino, Russia

2 “AquaLogo” group company, Moscow, Russia

*e-mail: orlovmikhailanat@gmail.com

Key words: Cyprinidae, SSRs, genomics, fish

Motivation and Aim: Short sequence repeats or SSRs are repeated DNA kinds with units 1 to 6 nucleotide long which are strictly adjacent. Length and sequence purity (e.g. lack of irregularities) were earlier found to collocate with a variety of genome hotspots, such as chromosome rearrangement loci or loci under strict selection. Here we consider SSR sets for Cyprinidae fish species. Length of SSRs as well as their oligonucleotide composition that we obtained differ clearly in domesticated vs free-living representatives. Cyprinidae are unique in their dramatic evolutionary history with complex polyploidy, distant hybridization as well as massive chromosome rearrangements events [1]. The family therefore is very well suited for studying SSRs evolution in general. Genes encoding traits under strict selection were shown to have SSRs length well correlated with the traits quantity [2]. SSRs are prone to mutate most rapidly, e.g. undergoing expansions (i.e. trinucleotide expansions) as well as contractions. These are connected with the rate of various evolutionary processes [3].

Methods and Algorithms: Here we consider SSRs for 28 Cyprinidae fish (including most notable goldfish Carassius auratus) deposited in FishMicrosat database [4]. For each species SSRs set we derived length in base pair and nucleotide composition. Namely, di-, tri- and tetranucleotide frequency was obtained. The result was furtherly interrogated using Ward's method clusterization and principal component analysis (PCA).

Results: Rather expectedly, domesticated fish mostly clustered together. So did the free-living species. PCA reinforced clusterization results while also pointing to significant differences between two clusters in sequence. Indeed, TG/CA dinucleotide presented more in domesticated fish with smaller amounts of AG/TC dinucleotide. The fact agrees with previous data of TG being more mutagenic.

Conclusion: The hereby obtained results drive attention to the Cyprinidae including goldfish as a valuable study subject for the aim of SSRs connection to evolution as well as breeding. Preliminary data suggest notable patterns of longer TG-rich simple sequence repeats in domesticated species.

\section{References}

1. Boron A., Spoz A., Porycka K. et al. Comparative Cytogenetics. 2014, V. 8(3), P. 233-248.

2. Fondon J. W., Garner H. R. 2004, V. 101(52), P. 18058-18063.

3. Xie K. T., G. Wang, A. C. Thompson et al. Science. 2019. V. 363. P. 81-84

4. Nagpure N.S., Rashid I., Pati R. et al. BMC Genomics. 2013, V. 14, P. 630. 